\title{
Persistence Analysis on Pre-coalition Models of H1N1-p with H5N1 virus in $L^{2}$ Space
}

\author{
Hariyanto, Mahmud Yunus and Gusti Yuni Shinta Lestari
}

\begin{abstract}
Influenza virus, H1N1-p and H5N1, are dangerous viruses. Medium of virus transmissions is the interaction or contact between individuals. The virus transmission to other individuals is easy. This happens due to a new strain that occurs as a result of pre-coalition between the two viruses. That phenomena is formulated in the form of a pre-coalition model of the virus. From the original pre-coalition model, a reduction process is done such that the models can be analyzed easily. Furthermore, the reduced pre-coalition model will be analyzed (i.e. existence and uniqueness of solutions), so that the system of equations is said to be well-posed. Persistence analysis result shows that in an unstable condition, H1N1-p influenza virus is "strongly uniformly persistence" over the system under the assumption that the H5N1 influenza virus is in a steady state. A similar result is also true for the H5N1 influenza virus. The H5N1 virus is more pathogenic than the H1N1-P-p. This is indicated by the value of $\varepsilon_{0}$ in H5N1 virus is smaller than in H1N1-p virus, where $\varepsilon_{0}$ shows the distance of interactions between individuals.
\end{abstract}

Index Terms-Persistence analysis, pre-coalition models, H1N1-p, H5N1, $L^{2}$ space.

\section{INTRODUCTION}

$\mathbf{T}$ HE development of science and technology in the medical field does not guarantee people will be free from diseases. This is because the causes of disease that comes from bacteria and viruses also developed and evolved so that viruses and bacteria become resistant to drugs. In short, evolution can be interpreted as a change in the properties inherited from earlier species. One form of the virus is a reassortment of genetic evolutions. Reassortment is the exchange of genetic informations between segments/bits of the genome when cells are in-coinfected with segmented RNA virus. Basically, reassortment is the cause of the coalition. The coalition is a process of joining two species into a new species with different characteristics. One virus that is able to evolve with the fast is H1N1-p and H5N1.

Liu et al [1] states that the H5N1 virus is very easily coalesced with H1N1-p virus if both viruses are transmitting on the same host. In addition, the dynamic mobility of individual residents can expand the ranges of H1N1-p and H5N1. Thus, there is a possibility of coalition between the $\mathrm{H} 5 \mathrm{~N} 1$ virus with the H1N1-p virus.

Hariyanto [2] constructed a mathematical model to describe a pre-coalition process between H1N1-p and H5N1 in Indonesia in $L^{1}$ space. The occurrence of pre-coalition between

Manuscript received August 11, 2016; accepted August 21, 2016.

The authors are with the Department of Mathematics, Institut Teknologi Sepuluh Nopember, Surabaya 60111, Indonesia. Emails: $\quad$ hariyanto, yunusm\}@matematika.its.ac.id, gusti.yunishinta@gmail.com the two viruses can be seen from their relationship with the existence of the reproduction number [3] of viruses in the system (persistence). In this paper, we reduce the virus model similar to [2], as well as looking at properties in the $L^{2}$ space [4]. Finally, we discuss whether the virus phenomenon that occurred in the study is also applicable in $L^{2}$ space.

\section{Mathematical Model of Pre-Coalition between H1N1-P AND H5N1 VIRUS}

In general, the spreading model of influenza virus H1N1-p and $\mathrm{H} 5 \mathrm{~N} 1$ at locations 1 and 2 can be expressed as follows [2]:

$$
\begin{aligned}
\frac{\partial S_{j m}}{\partial t}= & D_{j}^{S} \frac{\partial^{2} S_{j m}}{\partial x^{2}}-\beta^{*} S_{j m} I_{2 j u}-\alpha S_{j m} I_{1 j m}-d S_{j m} \\
& +\int_{\Omega_{k}} S_{k m} K(x-y) d y+b S_{j m} \\
& +S_{j m} \int_{\Omega_{j}} K(y-x) d x+\delta I_{2 j m}+\delta I_{1 j m}+\delta E_{1 j m}
\end{aligned}
$$

$$
\begin{aligned}
\frac{\partial E_{1 j m}}{\partial t}= & D_{1 j}^{E} \frac{\partial^{2} E_{1 j m}}{\partial x^{2}}+\alpha S_{1 j m} I_{1 j m}-\gamma E_{1 j m}-d E_{1 j m} \\
& +\int_{\Omega_{k}} E_{1 k m} K(x-y) d y-b E_{1 j m} \\
& +E_{1 j m} \int_{\Omega_{j}} K(y-x) d x-\delta E_{1 j m} \\
\frac{\partial I_{i j m}}{\partial t}= & \left(D_{1 j}^{I}+D_{2 j}^{I}\right) \frac{\partial^{2} I_{i j m}}{\partial x^{2}}+\gamma E_{1 j m}+\beta^{*} S_{j m} I_{2 j u} \\
& -d I_{i j m}-b I_{i j m}-\delta I_{i j m} \\
\frac{\partial S_{2 j u}}{\partial t}= & D_{2 j}^{S} \frac{\partial^{2} S_{2 j u}}{\partial x^{2}}-\beta S_{2 j u} I_{2 j u}-d S_{2 j u}+b S_{2 j u} \\
& +\int_{\Omega_{k}} S_{2 k u} K(x-y) d y+S_{2 j u} \int_{\Omega_{j}} K(y-x) d x
\end{aligned}
$$

$$
\frac{\partial I_{2 j u}}{\partial t}=D_{2 j}^{I} \frac{\partial^{2} I_{2 j u}}{\partial x^{2}}+\beta S_{2 j u} I_{2 j u}-d I_{2 j u}-b I_{2 j u}
$$

Index states $i$ represents the index of influenza viruses, where $i=1$ denotes H1N1-p influenza virus and $i=2$ denotes the H5N1 influenza virus. While the index $j$ represents the location, where $j=1$ denotes location 1 and $j=2$ denotes location 2. If the influenza viruses occur in humans, this is denoted by index $m$. If the spread of H5N1 influenza virus is in aves, we denote this by index $u$. Then index $k$ denotes the global diffusion between locations. If the spread of the virus occurs at the location $j=1$, global diffusion occurs in $k=2$. 


\section{DISCUSSION AND RESULTS}

\section{A. Mathematical Model}

In this section, we will reduce the pre-coalition model of $\mathrm{H} 5 \mathrm{~N} 1$ and H1N1-p based on changes in the virus as well as on each individual subpopulations. The purpose of the reduction of pre-coalition model of this virus is that the reduced model is easier to analyze. Before the reduction in pre-coalition models, we will clarify some notions in advance so that the coalition model can be reduced more efficiently, i.e. reducing the most significant part only.

Considering the assumption in the preceding paragraph, we can express the mathematical model of pra-coalition virus H1N1-p with H5N1 as follows.

1) Reduction of mathematical model of H1N1-p virus in humans in location 1

$$
\begin{aligned}
\frac{\partial S_{11 m}}{\partial t}= & D_{1}^{S} \frac{\partial^{2} S_{11 m}}{\partial x^{2}}-k E_{11 m} \\
& -\left(d-b+\mu-q_{1}-q_{2}\right) S_{11 m}+\mu S_{12 m} \\
\frac{\partial E_{11 m}}{\partial t}= & D_{11}^{E} \frac{\partial^{2} E_{11 m}}{\partial x^{2}}-(-k+d+b+\mu+n) E_{11 m} \\
& -p I_{11 m}+\mu \sigma E_{12 m} \\
\frac{\partial I_{11 m}}{\partial t}= & D_{11}^{I} \frac{\partial^{2} I_{11 m}}{\partial x^{2}}-(-p+d+b+v) I_{11 m}
\end{aligned}
$$

2) Reduction of mathematical model of $\mathrm{H} 5 \mathrm{~N} 1$ virus in humans in location 1

$$
\begin{aligned}
\frac{\partial S_{21 m}}{\partial t}= & D_{1}^{S} \frac{\partial^{2} S_{21 m}}{\partial x^{2}}-a I_{21 m} \\
& -(d-b+\mu-o) S_{21 m}+\mu S_{22 m} \\
\frac{\partial I_{21 m}}{\partial t}= & D_{21}^{I} \frac{\partial^{2} I_{21 m}}{\partial x^{2}}-(-a+d+b+u) I_{21 m}
\end{aligned}
$$

3) Reduction of mathematical model of $\mathrm{H} 5 \mathrm{~N} 1$ virus in aves in location 1

$$
\begin{aligned}
\frac{\partial S_{21 u}}{\partial t}= & D_{21}^{S} \frac{\partial^{2} S_{21 u}}{\partial x^{2}}-s I_{21 u}-(d-b+\mu) S_{21 u} \\
& +\mu S_{22 u} \\
\frac{\partial I_{21 u}}{\partial t}= & D_{21}^{I} \frac{\partial^{2} I_{21 u}}{\partial x^{2}}-(-s+d+b) I_{21 u}
\end{aligned}
$$

\section{B. Desoer Assumption}

The pre-coalition model of H1N1-p and H5N1 virus that has been reduced must be shown that the model satisfies the existence and uniqueness of solutions. We use the Desoer assumption to show the existence and uniqueness of the model. We do this before analyzing the persistence of the model. To demonstrate the existence and uniqueness of pre-coalition model of virus, we use Lipschitz constants.

\section{Persistence Analysis in $L^{2}$ Space}

In this section, we analyze the persistence of H1N1-p and $\mathrm{H} 5 \mathrm{~N} 1$ virus. This persistence analysis was conducted to determine the effect of the virus in the system. Analysis of persistence can be made only after a qualitative analysis on the reduced form of models: show the model has a positive solution that is unique. In the process, we calculate the value of $\varepsilon$ used in the analysis of the persistence of analytical solutions.

The mathematical model of pra-coalition is defined in (6)(12). By using the definition of persistence in [2], [5], we obtain:

1) Persistence of H1N1-p in human

- For $R_{01}<1$

$$
\begin{aligned}
& \limsup _{t \rightarrow \infty} d\left(S_{11 m}, I_{11 m}\right) \\
& =\sqrt{\frac{2}{\pi}}\left(\int_{\Omega_{1}}\left|\frac{1}{2} \sigma-I_{11 m 0}\right|^{2} d x\right)^{1 / 2} \\
& =\sqrt{\frac{2}{\pi} L_{1}}\left(\frac{1}{2} \sigma-I_{11 m 0}\right)
\end{aligned}
$$

Thus, there exists an $\varepsilon_{0}=\sqrt{\frac{2}{\pi} L_{1}}\left(\frac{1}{2} \sigma-I_{11 m 0}\right)>$ 0 such that the persistence of H1N1-p virus on the system in a stable condition is weakly uniform persistence. This means the transmission of H1N1$\mathrm{p}$ virus in humans occurs after contact between individual subpopulations susceptible and subpopulations infected. With $d\left(S_{11 m}, I_{11 m}\right)$ is the representation of the change in the number of infected or susceptible subpopulations. At the steady state condition, the system is weakly persistence, the contact between the individual and the local environments produces maximum distance.

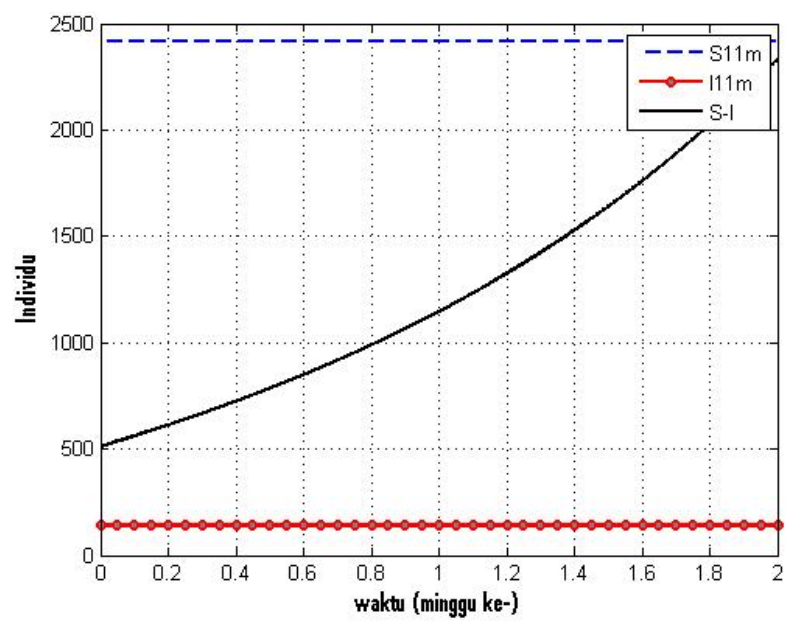

Fig. 1. Persistence of H1N1-p in human population when $R_{01}<1$.

We can see persistence when $R_{01}<1$ in Fig. 1 . $R_{01}<1$ is a condition where the system is in a stable condition. A condition in which the average individual who is infected can infect at most one individual. Thus, the existence of virus in the system is in a weakened state (weakly persistence). The persistence is very influential on the contact's distance between the individual. The farther the distance, the smaller the individual had contacted the disease. The smaller the distance, the greater the infected subpopulation $S$, and vice versa. In Fig. 1, it appears 
that the persistence is approaching subpopulations $S$. This approach means that the resilience of the virus is weakened so it approaches the graph of $S$ which means that many individuals are recovered. This is the condition of weakly uniform persistence.

- For $R_{01}>1$

$$
\begin{aligned}
& \liminf _{t \rightarrow \infty} d\left(S_{11 m}, I_{11 m}\right)=\sqrt{\frac{2}{\pi}} \\
& \left(\int_{\Omega_{1}}\left|\left(K_{1}-\frac{1}{2} K_{2}\right) \sigma-\frac{1}{2} K_{2} \sigma-K_{3} I_{11 m 0}\right|^{2} d x\right)^{1 / 2} \\
& =\sqrt{\frac{2 L_{1}}{\pi}}\left(\left(K_{1}-K_{2}\right) \sigma-K_{3} I_{11 m 0}\right)
\end{aligned}
$$

Thus there exists an $\varepsilon_{0}=\sqrt{\frac{2 L_{1}}{\pi}}\left(\left(K_{1}-K_{2}\right) \sigma-\right.$ $\left.K_{3} I_{11 m 0}\right)>0$ such that the persistence of $\mathrm{H} 1 \mathrm{~N} 1-$ $\mathrm{p}$ virus on the system in an unstable condition is strongly uniform persistence. This means to infect, the contact between the individual produces minimum distance. We can see persistence when $R_{01}>1$ on Fig. 2 .

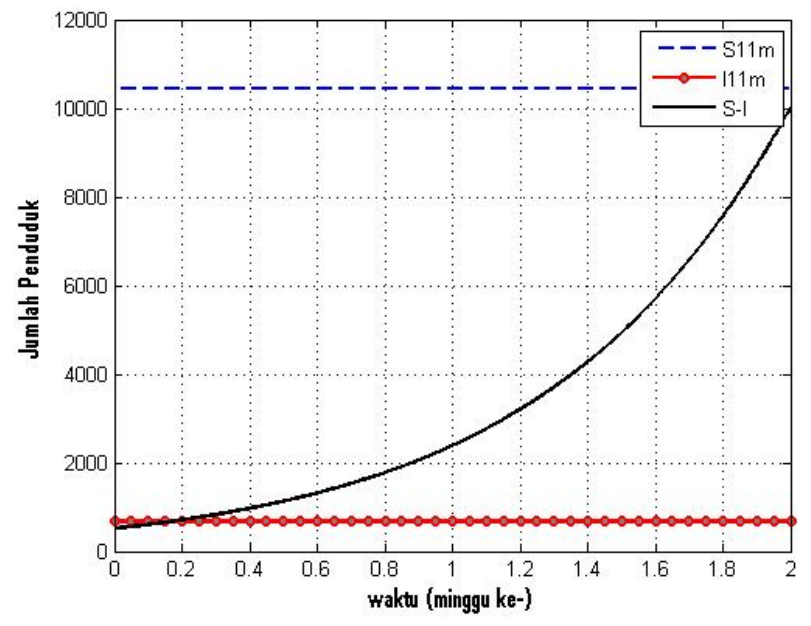

Fig. 2. Persistence of H1N1-p in human population when $R_{01}>1$.

$R_{01}>1$ is the condition where the infected individual is capable of infecting at least one individual. From Fig. 2, we know that the persistence is represented by a black graph initially approached the red graph. That means many people are infected, so the virus is said to be strongly uniform persistence.

2) Persistence of H5N1 in human

- For $R_{02}<1$

$$
\begin{aligned}
& \limsup _{t \rightarrow \infty} d\left(S_{21 m}, I_{21 m}\right) \\
& =\sqrt{\frac{2}{\pi}}\left(\int_{\Omega}\left(\frac{1}{2} \sigma-\sqrt{\frac{\pi}{2}} I_{21 m 0}\right)^{2} d x\right)^{1 / 2} \\
& =\sqrt{\frac{2}{\pi} L_{1}}\left(\frac{1}{2} \sigma-\sqrt{\frac{\pi}{2}} I_{21 m 0}\right)
\end{aligned}
$$

Thus there exists an $\varepsilon_{0}$ =
$\sqrt{\frac{2}{\pi} L_{1}}\left(\frac{1}{2} \sigma-\sqrt{\frac{\pi}{2}} I_{21 m 0}\right)>0$ such that the persistence of $\mathrm{H} 5 \mathrm{~N} 1$ virus on the system in a stable condition is weakly uniform persistence.

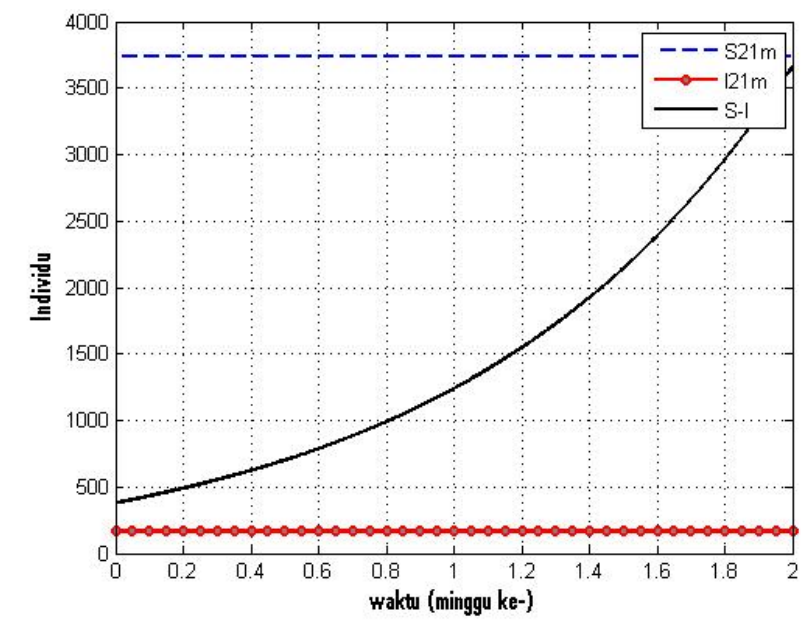

Fig. 3. Persistence of H5N1 in human population when $R_{02}<1$.

Figure 3 shows a simulation of the persistence of $\mathrm{H} 5 \mathrm{~N} 1$ for human when $R_{02}<1$. Persistence is represented as a graph with black color. As we can see from the graph, the persistence goes toward susceptible subpopulation. But there is a small difference compared to Fig. 1, which is more inclined to the black graphics representing infected subpopulation. It can be concluded also that H5N1 is more virulence than H1N1-p in human.

- For $R_{02}>1$

$$
\begin{aligned}
& \liminf _{t \rightarrow \infty} d\left(S_{21 m}, I_{21 m}\right)=\sqrt{\frac{2}{\pi}} \\
& \left(\int_{\Omega}\left(\left(M_{1}-M_{2}\right) \sigma-I_{21 m 0} \sqrt{\frac{\pi}{2}} M_{3}\right)^{2} d x\right)^{1 / 2} \\
& =\sqrt{\frac{2}{\pi} L_{1}}\left(\left(M_{1}-M_{2}\right) \sigma-I_{21 m 0} \sqrt{\frac{\pi}{2}} M_{3}\right)
\end{aligned}
$$

Thus there exists an $\varepsilon_{0}$ = $\sqrt{\frac{2}{\pi} L_{1}}\left(\left(M_{1}-M_{2}\right) \sigma-I_{21 m 0} \sqrt{\frac{\pi}{2}} M_{3}\right)>0$ such that the persistence of the H5N1 virus for human on the system in an unstable condition is strongly uniform persistence (see Fig. 4).

3) Persistence of H5N1 in aves

- For $R_{03}<1$

$$
\begin{aligned}
& \limsup _{t \rightarrow \infty} d\left(S_{21 u}, I_{21 u}\right)=\sqrt{\frac{2}{\pi}} \\
& \left(\int_{\Omega}\left|\left(1+\sqrt{\frac{2}{\pi}} S_{u 0}-P_{3} I_{u 0}\right)\right|^{2} d x\right)^{1 / 2}
\end{aligned}
$$

- For $R_{03}>1$ 


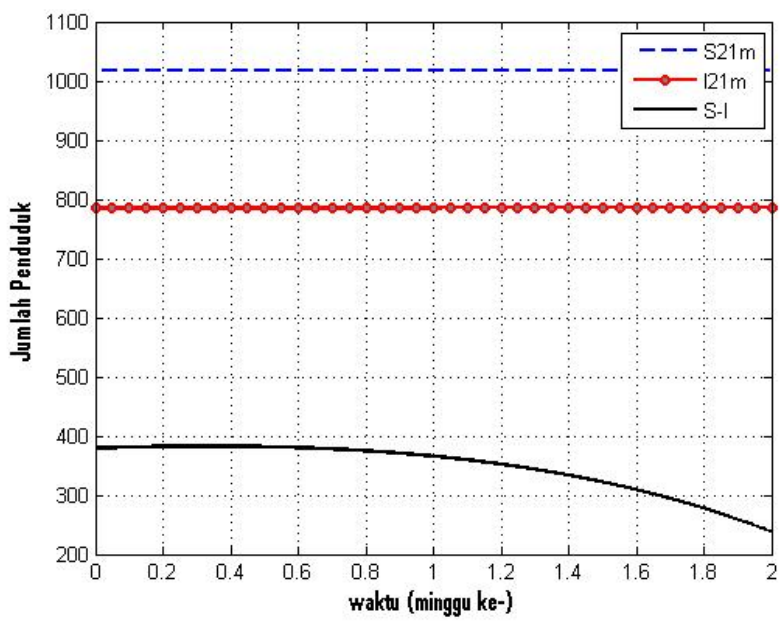

Fig. 4. Persistence of H5N1 in human population when $R_{02}>1$.

Thus there exists an $\varepsilon_{0}$ = $\left(\int_{\Omega}\left|\left(1+\sqrt{\frac{2}{\pi}} S_{u 0}-P_{3} I_{u 0}\right)\right|^{2} d x\right)^{1 / 2}>0$ such that the persistence of the H5N1 virus for aves on the system in an unstable condition is weakly uniform persistence (see Fig. 5).

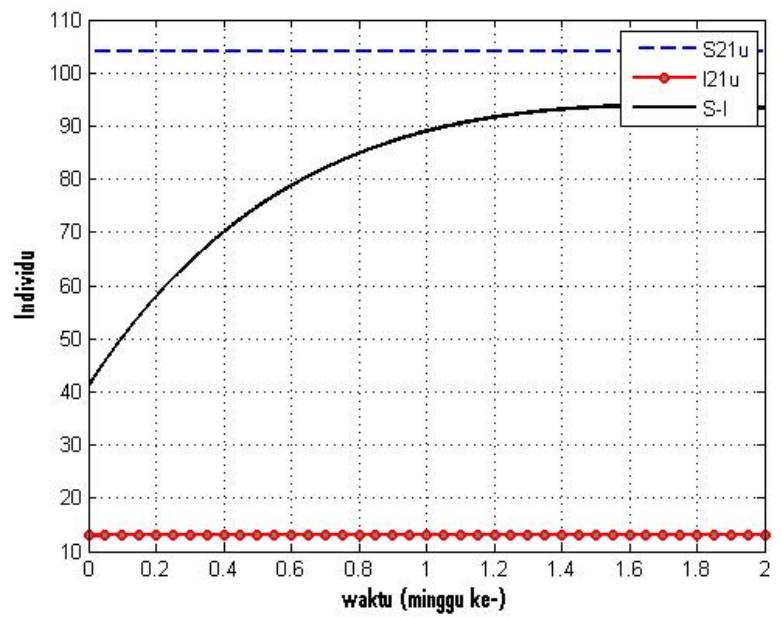

Fig. 5. Persistence of H5N1 in aves population when $R_{03}<1$.

Similar with Fig. 1 and Fig. 3, it appears that the persistence (black line) is approaching subpopulations $S$.

$$
\begin{aligned}
& \liminf _{t \rightarrow \infty} d\left(S_{21 u}, I_{21 u}\right)=\sqrt{\frac{2}{\pi}} \\
& \left(\int_{\Omega}\left|\left(P_{1}+\sqrt{\frac{2}{\pi}} P_{2}\right) S_{u 0}-P_{3} I_{u 0}\right|^{2} d x\right)^{1 / 2} \\
& =\sqrt{\frac{2}{\pi} L_{1}}\left(\left(P_{1}+\sqrt{\frac{2}{\pi}} P_{2}\right) S_{u 0}-P_{3} I_{u 0}\right)
\end{aligned}
$$

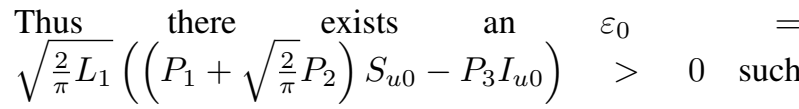

that the persistence of the H5N1 virus for aves on the system in an unstable condition is strongly uniform persistence (see Fig. 6).

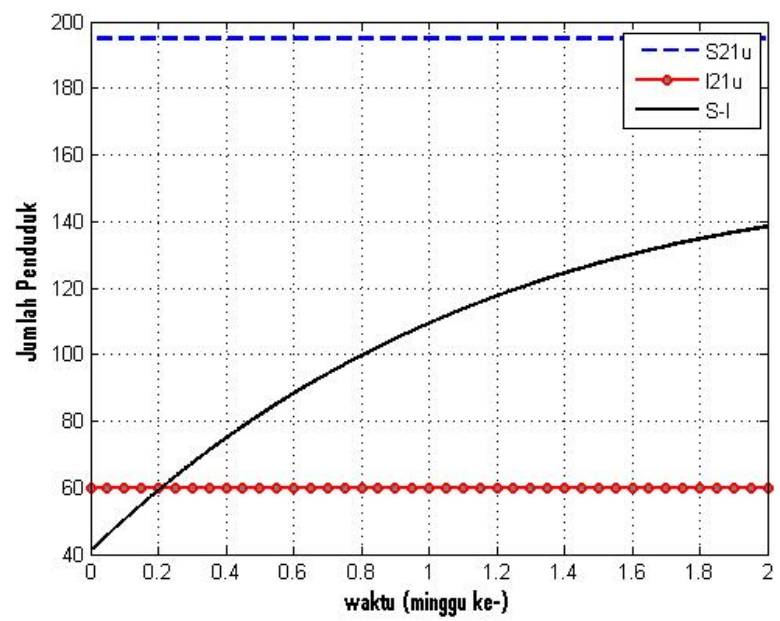

Fig. 6. Persistence of $\mathrm{H} 5 \mathrm{~N} 1$ in aves population when $R_{03}>1$.

\section{CONCLUSiOnS}

The reduction in pre-coalition models of virus is done in three stages: transmission of the virus, recovery and the ability of individuals to cast. In the unstable condition, the H1N1-p influenza virus is suffered strongly uniformly persistence of the system on the assumption that the H5N1 influenza virus is in a steady state. A similar situation occurs in the $\mathrm{H} 5 \mathrm{~N} 1$ influenza virus. The H5N1 virus is more pathogenic than the H1N1-p. This is indicated in the value of $\varepsilon_{0}$ of H5N1 is smaller than the value of $\varepsilon_{0}$ of H1N1-p, which shows the distance of interactions between individuals .

\section{REFERENCES}

[1] J. Liu, H. Xiao, F. Lei, Q. Zhu, K. Qin, X.-w. Zhang, X.-1. Zhang, D. Zhao, G. Wang, Y. Feng, J. Ma, W. Liu, J. Wang, and G. F. Gao, "Highly pathogenic h5n1 influenza virus infection in migratory birds," Science, vol. 309 , no. 5738, pp. 1206-1206, 2005.

[2] Hariyanto, B. Widodo, I. N. Budiantara, and C. Nidom, "The construction of a model of pre-coalition between $\mathrm{h} 1 \mathrm{n} 1-\mathrm{p}$ and $\mathrm{h} 5 \mathrm{n} 1$ influenza virus in Indonesia," Applied Mathematical Sciences, vol. 7, no. 99, pp. 4899-4907, 2013.

[3] P. Van den Driessche and J. Watmough, "Reproduction numbers and sub-threshold endemic equilibria for compartmental models of disease transmission," Mathematical Biosciences, vol. 180, no. 1, pp. 29-48, 2002

[4] R. Susanto, Jurnal Matematika, vol. 10, no. 3, pp. 91-97, 2007

[5] K. Das and D. Mukherjee, "Persistence aspects of an epidemic model of chagas disease," Tamsui Oxford Journal of Mathematical Sciences, vol. 25, no. 3, pp. 301-311, 2009. 